\title{
Confined swirling jets with large expansion ratios
}

\author{
By A. REVUELTA ${ }^{1}$, A. L. SÁNCHEZ ${ }^{2}$ AND A. LIÑ́N ${ }^{3}$ \\ ${ }^{1}$ Departamento de Combustibles Fósiles, CIEMAT, 28040 Madrid, Spain \\ ${ }^{2}$ Area de Mecánica de Fluidos, Universidad Carlos III de Madrid, 28911 Leganés, Spain \\ ${ }^{3}$ E. T. S. I. Aeronáuticos, Pl. Cardenal Cisneros 3, 28040 Madrid, Spain
}

(Received 16 December 2003 and in revised form 16 February 2004)

This paper presents the extension of our previous investigation of confined round jets with large Reynolds numbers and large expansion ratios (Revuelta, Sánchez \& Liñán $2002 a$ ) to the case of swirling jets with swirl numbers of order unity. In the absence of vortex breakdown, we encounter the four-region asymptotic structure identified earlier for the non-swirling jet, including a region of jet development where the azimuthal and axial velocity components are comparable. For the flow in the long recirculating eddy that forms downstream, where the pressure differences associated with the azimuthal motion become negligible, the jet is found to act as a point source with momentum flux equal to the flow force of the incoming jet, and angular momentum flux equal to that of the jet at the orifice. The solution for the weak circulation in this slender region, including the parameter-free leading-order description and the first-order corrections, is determined by integrating the azimuthal component of the momentum equation written in the boundary-layer approximation. The results are validated through comparisons with numerical integrations of the steady axisymmetric Navier-Stokes equations, which are also used to evaluate critical conditions for vortex breakdown.

\section{Introduction}

We consider the confined laminar jet formed when an incompressible fluid of density $\rho$ and kinematic viscosity $v$ flows through a pipe of radius $\varepsilon a$ into a much larger coaxial pipe of radius $a$. We shall assume that the jet Reynolds number $R e_{j}=$ $[J /(\pi \rho)]^{1 / 2} / \nu$, based on the jet momentum flux $J$ at the orifice, is much larger than unity, but still sufficiently small that the resulting steady slender jet remains stable. This configuration was investigated for the non-swirling jet by Revuelta, Sánchez \& Liñán (2002a, henceforth denoted as RSL), in the distinguished double limit in which the value of the Reynolds number $R e_{j} \varepsilon$ for the final Poiseuille flow is of order unity. This previous paper identified the four regions sketched in figure 1: a slender jet region $\mathrm{J}$ of characteristic length $\operatorname{Re}_{j} \varepsilon a$ where the jet develops as a free jet with constant momentum flux $J$, a surrounding outer region $\mathrm{O}$ of slow motion driven by the jet entrainment, a main flow region $\mathrm{M}$ of characteristic length $R e_{j} a$ where the jet exchanges momentum with the outer recirculating flow, and a final transition region $\mathrm{T}$ of characteristic length $R_{j} \varepsilon a$ to the final Poiseuille flow.

The present paper extends our previous analysis by considering the case of swirling jets. The swirl ratio $S=L /(J \varepsilon a)$, constructed with the initial flux of angular momentum, $L$, will be assumed to be of order unity, so that the azimuthal and axial velocity components are initially comparable in the slender region of jet development, and may lead to vortex breakdown as the value of $S$ is increased to a critical value $S_{c}$. A number of papers, including Hall (1972), Leibovich (1978), Escudier (1987), and Shtern \& Hussain (1999), have reviewed the topic of vortex breakdown in both 


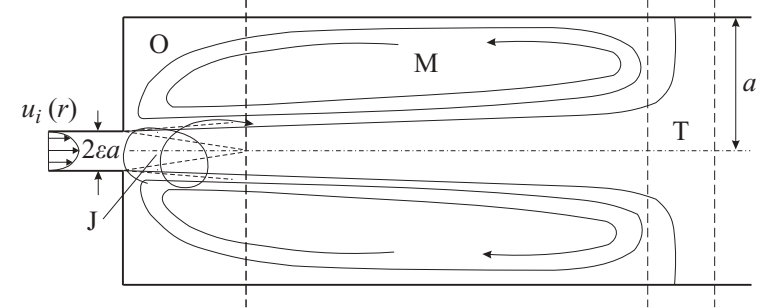

Figure 1. A schematic of the confined swirling jet flow.

confined and unconfined flows. The swirling-jet configuration considered here is relevant to combustion applications such as the dump combustor, for which the onset of breakdown is key for flame stabilization (see e.g. Poinsot \& Veynante 2001).

The main objective of the present study is the description of the flow structure found in the absence of vortex breakdown, i.e. for $S<S_{c}$, when we encounter the four regions identified for the non-swirling jet in RSL. In the slender jet that develops in region J, not affected by the outer confinement, the radial pressure differences associated with the azimuthal motion are of the order of the jet dynamic pressure. The induced adverse pressure gradient decelerates the jet significantly as it develops, reducing its momentum flux from the initial value $J$ to a final value equal to the jet flow force $M$, while the flux of angular momentum remains constant. On the other hand, the radial pressure differences associated with the azimuthal motion become negligible outside the region of jet development. Consequently, the radial and axial motion reduce in the first approximation to those induced by a swirl-free point source of momentum with momentum flux $M<J$, so that the results of RSL can be conveniently rescaled to provide the corresponding flow description. The weak swirling motion is determined a posteriori by integrating the azimuthal component of the momentum equation, with the jet acting in this case as a point source of angular momentum flux $L$, equal to the value at the orifice. The values of $M$ and $L$ are used for the description of the circulation in region $\mathrm{M}$, which reduces at leading order to a parameter-free problem independent of the shape of the velocity and circulation profiles at the orifice. The analysis determines the circulation distributions as the boundary regions $\mathrm{O}$ and $\mathrm{T}$ are approached, which are self-similar solutions of the second kind in which the exponents for the power-law decay with axial distance are obtained as eigenvalues for the two different local problems that appear. These asymptotic and local solutions are relevant for the study of jet precession, a phenomenon frequently observed in flows in burners, cyclone separators and spray dryers (Nathan, Hill \& Luxton 1998; Nathan, Turns \& Bandaru 1996). In particular, the description of the steady flow field in the different regions constitutes the base flow to enable the linear stability analysis in the limit configuration of large expansion ratios, which could provide limiting values for the oscillation frequencies, additional to those determined by numerical simulations (Guo, Langrish \& Fletcher 2001 $a, b$ ). The results given below are validated by performing integrations of the steady, axisymmetric, Navier-Stokes (NS) equations, which are also used to explore the onset of vortex breakdown in confined swirling jets with large expansion ratios.

\section{Formulation}

To write the governing equations in dimensionless form it is convenient to use as scales those corresponding to the main region, which are constructed with the initial 
fluxes of momentum and angular momentum $J$ and $L$. Thus, we define dimensionless axial and radial velocity components $v=v^{\prime} /(v / a)$ and $\hat{u}=u^{\prime} /\left[J /\left(\pi \rho a^{2}\right)\right]^{1 / 2}$, along with a dimensionless pressure $\hat{p}=p^{\prime} /\left[J /\left(\pi a^{2}\right)\right]$. The swirling motion is measured with the circulation of the azimuthal velocity $\Gamma^{\prime}=2 \pi r^{\prime} u_{\theta}^{\prime}$, which is scaled with its characteristic value $L /\left[\rho J a^{2} /(4 \pi)\right]^{1 / 2}$ to give the variable $\hat{\Gamma}$. The associated NS equations take the form

$$
\begin{aligned}
\frac{\partial \hat{u}}{\partial \hat{x}}+\frac{1}{r} \frac{\partial(r v)}{\partial r} & =0 \\
\hat{u} \frac{\partial \hat{u}}{\partial \hat{x}}+v \frac{\partial \hat{u}}{\partial r} & =-\frac{\partial \hat{p}}{\partial \hat{x}}+\frac{1}{r} \frac{\partial}{\partial r}\left(r \frac{\partial \hat{u}}{\partial r}\right)+\frac{1}{R e_{j}^{2}} \frac{\partial^{2} \hat{u}}{\partial \hat{x}^{2}} \\
\hat{u} \frac{\partial v}{\partial \hat{x}}+v \frac{\partial v}{\partial r} & =R e_{j}^{2}\left\{\varepsilon^{2} S^{2} \frac{\hat{\Gamma}^{2}}{r^{3}}-\frac{\partial \hat{p}}{\partial r}\right\}+\frac{\partial}{\partial r}\left(\frac{1}{r} \frac{\partial(r v)}{\partial r}\right)+\frac{1}{R e_{j}^{2}} \frac{\partial^{2} v}{\partial \hat{x}^{2}} \\
\hat{u} \frac{\partial \hat{\Gamma}}{\partial \hat{x}}+v \frac{\partial \hat{\Gamma}}{\partial r} & =r \frac{\partial}{\partial r}\left(\frac{1}{r} \frac{\partial \hat{\Gamma}}{\partial r}\right)+\frac{1}{R e_{j}^{2}} \frac{\partial^{2} \hat{\Gamma}}{\partial \hat{x}^{2}}
\end{aligned}
$$

where $r$ and $\hat{x}$ represent the radial and axial distances from the orifice centre scaled with $a$ and $\operatorname{Re}_{j} a$. The boundary conditions at $\hat{x}=0$ are

$$
\begin{gathered}
0 \leqslant r \leqslant \varepsilon: \quad \hat{u}=\varepsilon^{-1} U_{i}, \quad v=0, \hat{\Gamma}=\varepsilon^{-1} \Gamma_{i}, \\
\varepsilon<r \leqslant 1: \quad \hat{u}=v=\hat{\Gamma}=0,
\end{gathered}
$$

where the flow perturbations arising in the pipe upstream from the exit orifice are not accounted for, an appropriate simplification for the values $R e_{j} \gg 1$ considered here. For $\hat{x}>0$ the solution must satisfy the regularity condition at the axis

$$
r=0: \quad \partial \hat{u} / \partial r=v=\hat{\Gamma}=0
$$

together with the non-slip condition on the confining duct

$$
r=1: \quad \hat{u}=v=\hat{\Gamma}=0,
$$

while far downstream the flow must approach the swirl-free Poiseuille profile

$$
\hat{x} \gg 1: \quad \hat{u}=2 q\left(1-r^{2}\right) \varepsilon, v=\hat{\Gamma}=0,
$$

with the constant $q=\int_{0}^{\varepsilon} 2 \varepsilon^{-2} r U_{i} \mathrm{~d} r$, of order unity, denoting the jet volume flux scaled with its characteristic value $\left(\pi \varepsilon^{2} a^{2} J / \rho\right)^{1 / 2}$. As can be seen, the solution depends on the three parameters $\varepsilon, R e_{j}$ and $S$, and on the form of the initial velocity and circulation profiles at the jet orifice, $U_{i}$ and $\Gamma_{i}$. The integrations performed below correspond to a jet of initial uniform velocity $U_{i}=1$ and with solid-body rotation $\Gamma_{i}=2(r / \varepsilon)^{2}$.

In the slender jet region $\mathrm{J}$, corresponding to axial distances of order $\operatorname{Re}_{j} \varepsilon a$ and to radial distances of order $\varepsilon a$, the flow is in the first approximation that of an unconfined swirling jet discharging into an infinite stagnant atmosphere. In the absence of vortex breakdown, the quasi-cylindrical approximation can be used to provide the velocity field, with relative errors of order $R e_{j}^{-2}$. The resulting equations can be written in terms of the rescaled variables of order unity, $r / \varepsilon, \hat{x} / \varepsilon, \varepsilon \hat{u}, \varepsilon v, \varepsilon^{2} \hat{p}$, and $\varepsilon \hat{\Gamma}$, by discarding the last term in (2.2) and (2.4) and retaining only the terms in curly brackets in (2.3). The problem was recently integrated numerically by Revuelta, Sánchez \& Liñán (2004) for $U_{i}=1$ and $\Gamma_{i}=2(r / \varepsilon)^{2}$. For values of $S$ above a critical value $S=0.64$, the integration fails to converge at a given downstream location, a failure that Hall (1972) associates with the emergence of vortex breakdown, a point supported by the 
numerical integrations of confined swirling flows in pipes performed by Beran \& Culick (1992) and Herrada, Pérez-Saborid \& Barrero (2003).

The velocity and circulation in the developing jet are seen to satisfy the conservation of angular momentum $\int_{0}^{r \gg \varepsilon} 2 r \hat{u} \hat{\Gamma} \mathrm{d} r=1$, together with the momentum balance equation

$$
\int_{0}^{r \gg \varepsilon} 2 r\left(\hat{p}+\hat{u}^{2}\right) \mathrm{d} r=m
$$

This last equation describes how the dimensionless momentum flux of the jet $\int_{0}^{r \gg \varepsilon} 2 r \hat{u}^{2} \mathrm{~d} r$ decreases from the initial value $\int_{0}^{\varepsilon} 2 r \hat{u}^{2} \mathrm{~d} r=1$ at the orifice to the final asymptotic value $\int_{0}^{r \gg \varepsilon} 2 r \hat{u}^{2} \mathrm{~d} r=m$ as the pressure perturbations become negligible in the far field, where the solution for the velocity is that given by Schlichting (1933) for the swirl-free jet:

$$
\hat{u}=\frac{m}{\hat{x}+\varepsilon X_{0}} \frac{512 / 3}{\left(64 / 3+\eta^{2}\right)^{2}},
$$

while the weak circulation is

$$
\hat{\Gamma}=\frac{1}{\hat{x}+\varepsilon X_{0}} \frac{16 \eta^{2}}{\left(64 / 3+\eta^{2}\right)^{2}},
$$

a result due to Görtler (1954) and Loitsianskii (1953). Here, $\eta=m^{1 / 2} r /\left(\hat{x}+\varepsilon X_{0}\right)$ represents the appropriate self-similar variable for the far-field description and the constant $X_{0}$ appearing as a first-order correction is the so-called virtual origin, to be computed from the numerical integration of the jet development region (Revuelta, Sánchez \& Liñán 2002b). The value of the dimensionless flow force $m=M / J=1+\int_{0}^{\varepsilon} 2 r \hat{p}_{i} \mathrm{~d} r$, giving the ratio of the momentum flux of the developed jet $M$ to the initial momentum flux $J$, can be computed by evaluating (2.10) at the orifice, where the radial pressure differences $\hat{p}_{i}=-S^{2} \int_{r}^{\varepsilon} \Gamma_{i}^{2} / r^{3} \mathrm{~d} r$ are determined from the radial momentum balance in terms of the initial circulation $\Gamma_{i}$, yielding for instance $m=1-S^{2}$ for solid-body rotation.

\section{Swirling motion in the main region $\mathbf{M}$}

We now proceed to study for $S<S_{c}$ the flow in the slender recirculating region $\mathrm{M}$ that extends downstream over distances of order $R e_{j} a$ from the jet orifice, where the motion is driven by the developed jet. This acts as a point source of momentum with angular momentum flux $L$, equal to that of the jet at the orifice, and with momentum flux $M$, smaller than the value $J$ at the jet orifice. The momentum flux $M$ of the developed jet is used as scale to define $x=m^{-1 / 2} \hat{x}, u=m^{-1 / 2} \hat{u}, \Gamma=m^{1 / 2} \hat{\Gamma}$ and $p=m^{-1} \hat{p}$ as appropriate variables for this region M. In the limit $R e_{j} \gg 1$ with $R e_{j} \varepsilon \sim 1$ equations (2.1) and (2.2) reduce, with relative small errors of order $R e_{j}^{-2} \sim \varepsilon^{2}$, to the boundary-layer (BL) equations, to be integrated for $u, v$ and $p$ independently of the circulation, which is determined by integrating

$$
u \frac{\partial \Gamma}{\partial x}+v \frac{\partial \Gamma}{\partial r}=r \frac{\partial}{\partial r}\left(\frac{1}{r} \frac{\partial \Gamma}{\partial r}\right)
$$

obtained from (2.4) at the same level of accuracy.

As explained in RSL, the boundary conditions for the velocity as $x \rightarrow 0$ arise from matching with the flow in the intermediate boundary region $\varepsilon \ll x \ll 1$, where the flow near the axis has developed to give the self-similar profile (2.11), whereas for $r \sim O(1)$ 

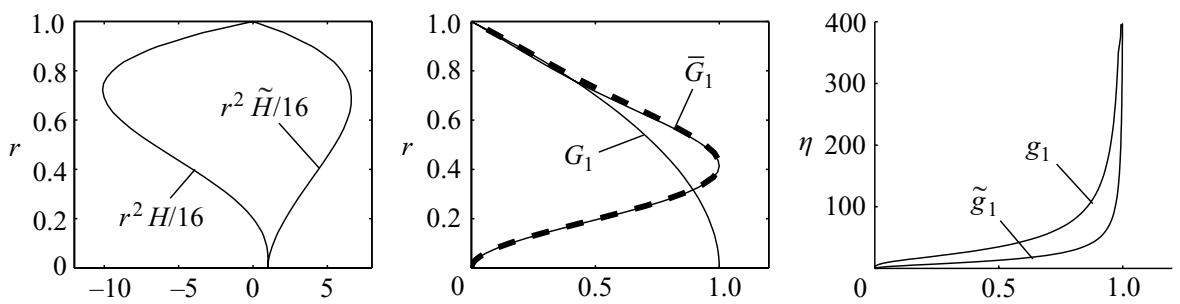

Figure 2. The functions $H(r), \tilde{H}(r), G_{1}(r), \bar{G}_{1}(r), g_{1}(\eta)$, and $\tilde{g}_{1}(\eta)$; the dashed line represents the radial distribution of the normalized circulation $\Gamma / \Gamma_{\max }$ determined at $x=0.177$ by numerical integration of (3.1).

the flow is directed towards the backstep with a local self-similar velocity profile $u=\left[x+m^{-1 / 2} \varepsilon\left(X_{0}-q / 8\right)\right] F^{\prime} / r$, with $F(r)$ representing an appropriately rescaled stream function. Integrating the BL equations with boundary conditions $u=v=0$ at $r=1$ and $\partial u / \partial r=v=0$ at $r=0$ for $x>0$ and with the initial profile $u=(512 / 3) /(x+$ $\left.m^{-1 / 2} \varepsilon X_{0}\right)^{-1}\left[64 / 3+r^{2} /\left(x+m^{-1 / 2} \varepsilon X_{0}\right)^{2}\right]^{-2}+\left[x+m^{-1 / 2} \varepsilon\left(X_{0}-q / 8\right)\right] F^{\prime} / r$ evaluated at $\varepsilon \ll x \ll 1$ provides, with errors of order $\varepsilon^{2}$, the solution for $u, v$ and $p$ in the main recirculating region. The solution at leading order, determined by setting $\varepsilon=0$ in the initial velocity profile, has a zero mass flux, and results in a closed eddy that ends at a stagnation plane located at $x=x_{s}$. The mass flux is accounted for in the first-order correction, which allows the flow to approach Poiseuille's solution $u=2 m^{-1 / 2} q\left(1-r^{2}\right)$ downstream.

\subsection{The initial circulation profile}

The weak swirling motion present in $\mathrm{M}$ is determined by integrating the linear equation (3.1). The integration uses as boundary condition the initial circulation profile appearing at $\varepsilon \ll x \ll 1$. The Gortler-Loitsiatskii jet solution (2.12), which appears near the axis, needs to be complemented with the solution at distances $r \sim O(1)$. The asymptotic behaviour $\Gamma \rightarrow 16 x / r^{2}$ of (2.12) for $r \gg x$ motivates the selection $\Gamma=\left[x+m^{-1 / 2} \varepsilon\left(X_{0}-q / 8\right)\right] H+m^{-1 / 2} \varepsilon(q / 8) \tilde{H}$ as the appropriate local solution describing the decay of the Gortler-Loitsiatskii circulation at distances $r$ of order unity. The function $H(r)$ and its first-order correction $\tilde{H}$, required to satisfy the matching with (2.12), are determined from the problems

$$
\begin{aligned}
H^{\prime \prime}+(F-1) H^{\prime} / r-F^{\prime} H / r=0 ; & H(1)=0, \quad H(r \rightarrow 0) \rightarrow 16 / r^{2}, \\
\tilde{H}^{\prime \prime}+(F-1) \tilde{H}^{\prime} / r=0 ; & \tilde{H}(1)=0, \quad \tilde{H}(r \rightarrow 0) \rightarrow 16 / r^{2},
\end{aligned}
$$

giving the solution shown in figure 2 .

According to the above description, the circulation decreases linearly with distance as the backstep is approached. The circulation surrounding the jet is however much larger, and is due to the swirling flow that recirculates to approach the backstep wall from region $\mathrm{M}$. To determine the solution, one needs to investigate the existence of eigensolutions of the form $\Gamma=C_{n}\left[x+m^{-1 / 2} \varepsilon\left(X_{0}-q / 8\right)\right]^{\lambda_{n}} G_{n}(r)$ where the eigenvalues $\lambda_{n}$ and accompanying eigensolutions $G_{n}(r)$ are determined from the eigenvalue problem

$$
G_{n}^{\prime \prime}+(F-1) G_{n}^{\prime} / r-\lambda_{n} F^{\prime} G_{n} / r=0, \quad G_{n}(1)=G_{n}^{\prime}(0)=0,
$$

while the constants of order unity $C_{n}$ are determined from matching with the numerical solution of the circulation field in M, as explained below. Non-trivial solutions of (3.4) exist for a discrete set of positive values $\lambda_{n}=0.43441,3.62063, \cdots$. The eigenfunction $G_{1}$ associated with the smallest eigenvalue is shown in figure 2 . The resulting profile 
is normalized to give a maximum value equal to unity at $r=0$. This outer solution is modified at distances $r \sim x$, where the circulation must decrease to reach the axis with a zero value. In this near-axis region the eigensolutions take the form $\Gamma=C_{n}\left[x+m^{-1 / 2} \varepsilon X_{0}\right]^{\lambda_{n}} g_{n}(\eta)-m^{-1 / 2} \varepsilon(q / 8) \lambda_{n} C_{n} x^{\lambda_{n}-1} \tilde{g}_{n}$, where the function $g_{n}(\eta)$ and its accompanying first-order correction $\tilde{g}_{n}$ are obtained from

$$
\begin{aligned}
g_{n}^{\prime \prime}+(f-1) g_{n}^{\prime} / \eta-\lambda_{n} f^{\prime} g_{n} / \eta=0 ; & g_{n}(0)=0, \quad g_{n}(\eta \rightarrow \infty) \rightarrow 1, \\
\tilde{g}_{n}^{\prime \prime}+(f-1) \tilde{g}_{n}^{\prime} / \eta-\left(\lambda_{n}-1\right) f^{\prime} \tilde{g}_{n} / \eta=0 ; & \tilde{g}_{n}(0)=0, \quad \tilde{g}_{n}(\eta \rightarrow \infty) \rightarrow 1,
\end{aligned}
$$

with $f=4 \eta^{2} /\left(64 / 3+\eta^{2}\right)$ and $\eta=r /\left(x+m^{-1 / 2} \varepsilon X_{0}\right)$ representing the stream function and the self-similar coordinate for Schlichting's jet solution (2.11), the prime denoting here differentiation with respect to this coordinate. The functions $g_{1}$ and $\tilde{g}_{1}$ associated with the first eigenvalue are shown in figure 2.

Note that the above self-similar solution for the flow that recirculates towards the backstep enters as boundary condition to determine the distribution of circulation in region $\mathrm{O}$ that surrounds the developing jet J. Since the pressure differences associated with the weak swirling motion are negligible in this region also, the axial and radial velocity components are those given in RSL for the non-swirling jet, while the circulation follows from integrating (2.4) written in terms of the rescaled variable $\Gamma_{o}=\Gamma /\left(C_{1} \varepsilon^{\lambda_{1}} / m^{\lambda_{1} / 2}\right)$, with $R e_{j} \varepsilon$ entering as the relevant local Reynolds number. The appropriate boundary conditions written in terms of $X=\hat{x} / \varepsilon$ are $\Gamma_{o}=0$ at $X=0, \Gamma_{o}=\left(X+X_{0}-q / 8\right)^{\lambda_{1}} G_{1}(r)$ as $X \rightarrow \infty$, and $\Gamma_{o}=0$ and $\partial \Gamma_{o} / \partial r=0$ at $r=1$ and $r \rightarrow 0$, respectively, for $0<X<\infty$. The integration would determine in particular the distribution of $\Gamma_{o}$ at the axis, corresponding to weak circulation of the fluid being entrained by the jet as it develops.

\subsection{Leading-order solution}

The leading-order description for the circulation in $\mathrm{M}$ is determined by integating numerically (3.1) with boundary conditions $\Gamma=0$ at $r=0$ and at $r=1$ for $x>0$ and with an initial condition obtained by evaluating the uniformly valid description

$$
\Gamma=\frac{16}{x^{2}} \frac{r^{2} / x^{2}}{\left(64 / 3+r^{2} / x^{2}\right)^{2}}+x\left(H(r)-16 / r^{2}\right)+C_{1} x^{\lambda_{1}}\left[G_{1}(r)+g_{1}(r / x)-1\right]
$$

at $x \ll 1$. As can be seen, using the fluxes $L$ and $M$ as scales for the different variables results in a parameter-free problem independent of the shape of the initial velocity and circulation profiles $U_{i}$ and $\Gamma_{i}$ at the orifice.

The equations were integrated with a pseudo-transient finite-volume scheme, described in detail in the appendix of RSL. The value of the constant $C_{1}=69.372$ was obtained as part of the integration by performing a least-square fit of (3.7) to the circulation profile obtained numerically at $x \ll 1$ at each time step. Isocontours of circulation are plotted in figure 3, along with the corresponding transverse profiles at five different locations. For completeness, the figure also shows the corresponding streamlines and profiles of axial velocity from RSL for the swirl-free jet. In comparing figure 3 with figure 7 of RSL one should bear in mind that the scaling used for $x$ here differs by a factor $\pi^{1 / 2}$ from that used in RSL, so that the resulting eddy length $x_{s}=0.188$ also differs by the same numerical factor.

As explained in RSL, the axial velocity near the rear stagnation plane $x=x_{s}$ decays linearly with distance, with a local similarity description of the form $u=-\left(x_{s}-x\right) \bar{F}^{\prime} / r$, where $\bar{F}$ is the similarity stream function. The profiles shown in figure 3 reveal that the decay of the circulation as the stagnation plane $x=x_{s}$ is approached is much faster. In seeking the corresponding local description for the circulation at $x_{s}-x \ll 1$ one 

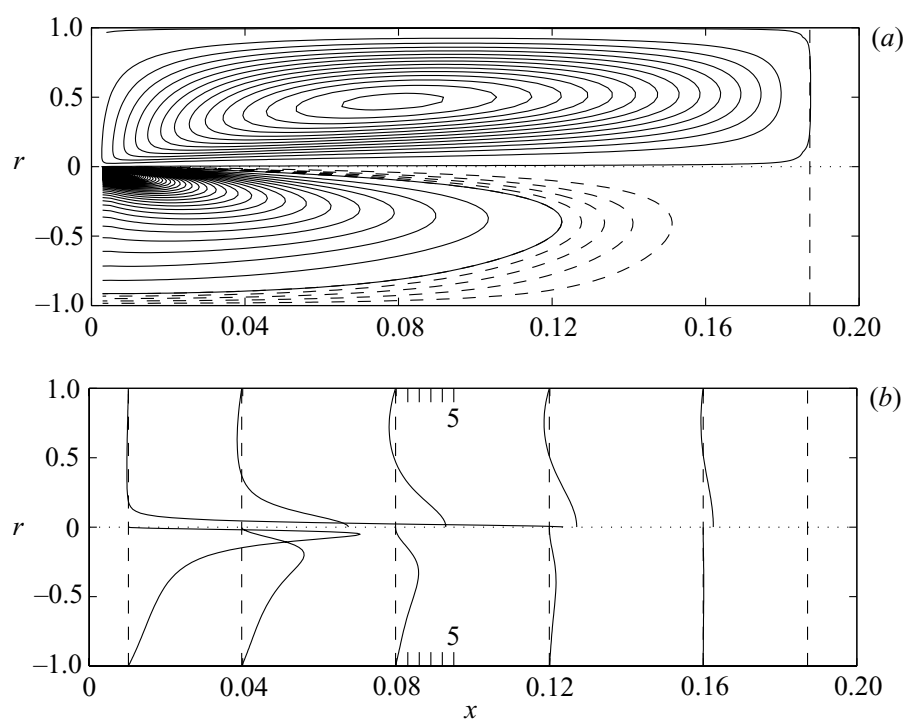

FIGURE 3. The leading-order solution for the confined swirling jet, including streamlines (upper half of $a$ ), isocontours of circulation (lower half of $a$ ), and velocity and circulation profiles at different downstream locations $(b)$.

needs to investigate the existence of eigensolutions of the form $\Gamma=\bar{C}_{n}\left(x_{s}-x\right)^{\bar{\lambda}_{n}} \bar{G}_{n}(r)$, where the eigenvalues $\bar{\lambda}_{n}$ and associated eigenfunctions $\bar{G}_{n}(r)$ are determined from the solution of

$$
\bar{G}_{n}^{\prime \prime}+(\bar{F}-1) \bar{G}_{n}^{\prime} / r+\bar{\lambda}_{n} \bar{F}^{\prime} \bar{G}_{n} / r=0, \quad \bar{G}_{n}(1)=\bar{G}_{n}(0)=0 .
$$

The eigenfunction corresponding to the smallest eigenvalue $\bar{\lambda}_{1}=2.4665$, normalized to give a maximum unity value, is shown in figure 2 . The value of the constant $\bar{C}_{1}=0.211$ was obtained by comparing the local solution $\Gamma=\bar{C}_{1}\left(x_{s}-x\right)^{\bar{\lambda}_{1}} \bar{G}_{1}(r)$ with the circulation profile obtained as $x \rightarrow x_{s}$ by numerical integration of (3.1). The comparison in figure 2 between the normalized circulation profile $\Gamma / \Gamma_{\max }$ obtained numerically at $x=0.177$ and the eigenfunction $\bar{G}_{1}$ constitutes a satisfactory test for the accuracy of the numerical scheme used in the integrations. Note that the eigensolution $\bar{G}_{1}$ would enter as appropriate boundary condition as $\left(x_{s}-x\right) / \varepsilon \rightarrow \infty$ when describing the circulation in the boundary region $\mathrm{T}$, where $\Gamma \sim \varepsilon^{\overline{\lambda_{1}}}$, in a development that parallels that outlined above for region $\mathrm{O}$.

\subsection{First-order correction}

As shown in RSL, corrections of order $\varepsilon$ can be accounted for in the BL computation of the velocity field by including the terms of order $\varepsilon$ in the initial condition for $u$. Similarly, the solution for the circulation field in region $\mathrm{M}$ can be computed with small relative errors of order $\varepsilon^{2}$ by using

$$
\begin{aligned}
\Gamma= & 16\left(x+m^{-1 / 2} \varepsilon X_{0}\right)^{-2} \eta^{2}\left(64 / 3+\eta^{2}\right)^{-2}+\left[x+m^{-1 / 2} \varepsilon\left(X_{0}-q / 8\right)\right]\left(H-16 / r^{2}\right) \\
& +m^{-1 / 2} \varepsilon(q / 8)\left(\tilde{H}-16 / r^{2}\right)+C_{1}\left[x+m^{-1 / 2} \varepsilon\left(X_{0}-q / 8\right)\right]^{\lambda_{1}}\left(G_{1}(r)-1\right) \\
& +C_{1}\left[x+m^{-1 / 2} \varepsilon X_{0}\right]^{\lambda_{1}} g_{1}(\eta)-m^{-1 / 2} \varepsilon(q / 8) \lambda_{1} C_{1} x^{\lambda_{1}-1} \tilde{g}_{1}(\eta)
\end{aligned}
$$

as the initial profile for $\Gamma$, to be evaluated at $\varepsilon \ll x \ll 1$. Note that the swirl number, $S$, and the shape of the circulation distribution at the orifice, $\Gamma_{i}$, enter in the first-order 

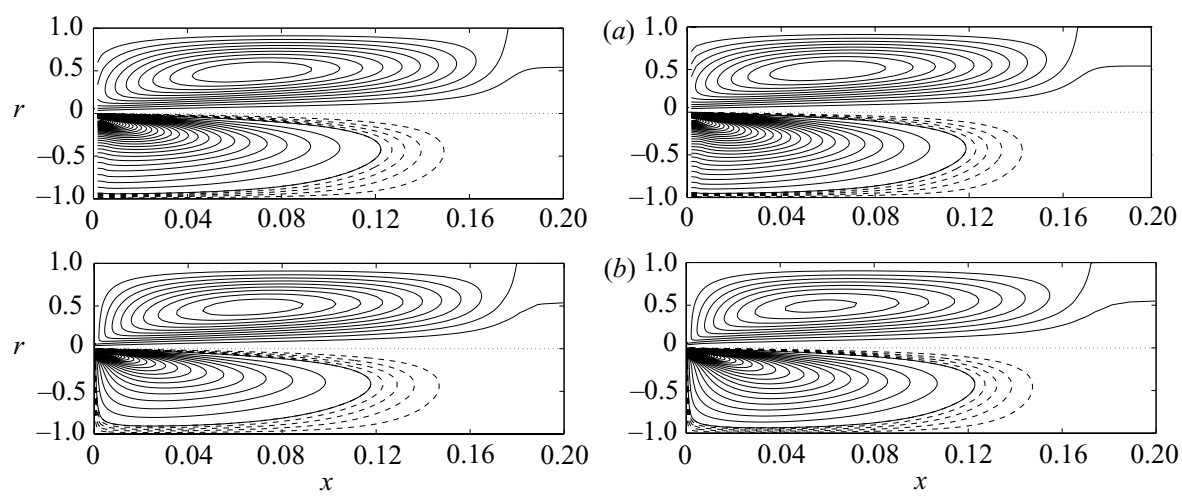

FIGURE 4. Streamlines and circulation isocontours obtained for $\varepsilon=0.05$ from the BL problem $(a)$ and from integration of the NS problem $(b)$ for $S=0.3$ (left-hand-side plots) and $S=0.6$ (right-hand-side plots).

correction for the solution in the main region through the values of $m$ and $X_{0}$, while the initial velocity distribution $U_{i}$ enters in the correction through the values of $m$ and $q$.

The solution for the resulting circulation isocontours for $\varepsilon=0.05$ is given in figure 4 for $S=(0.3,0.6)$. The values $m=1-S^{2}, q=1$ and $X_{0}=(0.271,0.410)$ for $S=(0.3,0.6)$ are employed in evaluating (3.9), corresponding to a uniform axial velocity with solidbody rotation (Revuelta et al. 2004). Since the momentum flux for the developed jet $M$, rather than the initial momentum flux $J$, is used in the scales for the different flow variables in this region $\mathrm{M}$, only small differences of order $\varepsilon$ are observed in the results.

To validate the asymptotic description, the NS axisymmetric problem given in (2.1)(2.9) with $U_{i}=1$ and $\Gamma_{i}=2(r / \varepsilon)^{2}$ was integrated numerically for different values of $R e_{j} \gg 1$ and $\varepsilon \ll 1$ and for different values of $S$. As in previous studies of swirling jets (see e.g. Ruith et al. 2003), the boundary condition given in (2.9) for the velocity components was replaced in the integrations by a radiation condition $\partial / \partial t+\hat{u} \partial / \partial \hat{x}=0$. The integrations were performed with two different algorithms, the SIMPLE algorithm (Patankar \& Spalding 1972) for the pseudo-transient problem and the projection method (Kim \& Moin 1985; Bell, Colella \& Glaz 1989) for the unsteady conservation equations, giving insignificant differences when the final steady state was achieved. For the sample results included in the comparisons of figure 4, the jet Reynolds number was selected to be $R e_{j}=(366.9,437.5)$ for $S=(0.3,0.6)$, thereby giving in both cases $[M /(\pi \rho)]^{1 / 2} / \nu=350$ for the effective Reynolds number based on the flow force $M$. To facilitate the comparison between the NS and the BL integrations, the results of the former are represented using the streamwise coordinate $x$. As can be seen, the degree of agreement is certainly satisfactory, with discrepancies becoming somewhat larger for $S=0.6$.

\section{Vortex breakdown in confined jets with large expansion ratios}

Although the above asymptotic description pertains to swirling jets with $S<S_{c}$, some conclusions can be drawn regarding the vortex breakdown behaviour of confined jets with $\varepsilon \ll 1$. Thus, we have seen that the pressure gradient induced by the swirling motion is only significant in region $\mathrm{J}$, whereas the azimuthal motion is very weak elsewhere. Hence, vortex breakdown must occur in region $\mathrm{J}$, at a critical value of the swirl number, $S_{c}$, equal in the first approximation to that of a free swirling jet 


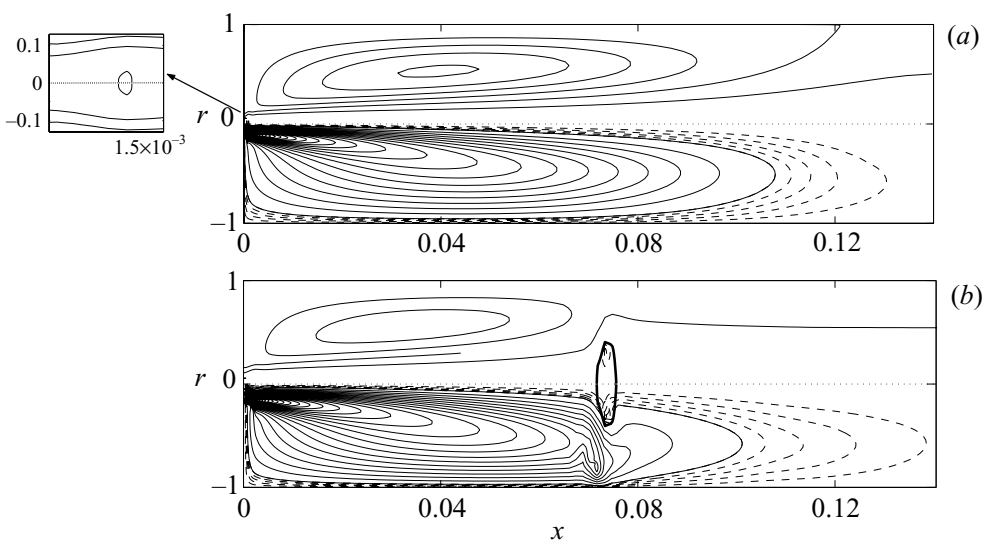

FIGURE 5. Streamlines and circulation isocontours obtained from the NS integrations with $[M /(\pi \rho)]^{1 / 2} / \nu=350$ and $S=0.68$ for $\varepsilon=0.10(a)$ and $\varepsilon=0.15(b)$; the inset in $(a)$ and the thick line in $(b)$ indicate the location of the recirculating bubble.

discharging into a stagnant atmosphere. The corrections to this leading-order result can be anticipated by noting that the confinement slightly perturbs the flow of the outer fluid being entrained by the jet, which is no longer stagnant when $\varepsilon \neq 0$. In particular, the non-zero circulation existing outside the jet when $\varepsilon \neq 0$ causes the jet flux of angular momentum to increase, thereby promoting breakdown for smaller values of $S_{c}$. The perturbations are small however, as seen above; the circulation of the fluid surrounding the jet in the region of jet development $x \sim \varepsilon$ is order $\varepsilon^{1+\lambda_{1}}$ smaller than that at the orifice, so that the relative decrease $S_{c}(\varepsilon=0)-S_{c}(\varepsilon)$ should be of that order of magnitude.

To test the above ideas, a series of numerical integrations of the axisymmetric NS equations was performed, providing the critical value of $S_{c}$ and the modified flow configuration emerging after breakdown for different values of $\varepsilon$. In the range of Reynolds number considered, $100 \leqslant R e_{j} \leqslant 500$, the results were seen to be independent of $R e_{j}$. According to the integrations, vortex breakdown like that of a free jet occurs only for sufficiently small values of $\varepsilon \lesssim 0.15$, leading to the appearance in the jet development region $\mathrm{J}$ of a recirculating bubble of size comparable to the orifice diameter. A sample of the resulting flow field is shown in figure 5(a). As can be seen, the presence of the small recirculating bubble reduces the jet momentum flux by a non-negligible amount, causing the toroidal vortex to be considerably shorter than that shown in figure 4 for $S<S_{c}$. The computations give $S_{c}=0.66 \pm 0.01$ for $0.01 \leqslant \varepsilon<0.15$, confirming that the influence of the outer confinement on the critical conditions for breakdown is very limited. It is of interest that the critical value obtained, $S_{c}=0.66$, is very close to the value $S=0.64$ reported by Revuelta et al. (2004) for the failure of the quasi-cylindrical integration of the free jet, indicating that the criterion of Hall (1972) predicts well the onset of breakdown in this case. As a final comment on this type of breakdown, note that the order of magnitude of the departures obtained, $S_{c}-0.66= \pm 0.01$, is in agreement with the prediction of the asymptotic analysis, $S_{c}(\varepsilon=0)-S_{c}(\varepsilon) \propto \varepsilon^{1+\lambda_{1}}$, although the degree of accuracy of the numerical scheme was not sufficient to determine the proportionally constant $\left[S_{c}(\varepsilon=0)-S_{c}(\varepsilon)\right] / \varepsilon^{1+\lambda_{1}}$. The computation of this proportionality factor by extending to a higher order the analysis of region $\mathrm{J}$, with account taken of the small relative circulation of order $\varepsilon^{1+\lambda_{1}}$ of the entrained fluid, does not seem to be amenable to a simple perturbation scheme. 
For values of $\varepsilon \gtrsim 0.15$ vortex breakdown no longer occurs in region $\mathrm{J}$, but rather at the rear end of the toroidal vortex, where the stream tube bounding the toroidal vortex opens up, yielding a recirculating bubble of size comparable to that of the confining duct. The resulting toroidal vortex is considerably shorter, as can be seen to occur for $\varepsilon=0.15$ in figure $5(b)$. The streamlines in the figure indicate that vortex breakdown is similar in this case to that encountered in pipes, with the separating stream tube acting in this case as a diverging pipe (Buntine \& Saffman 1995). The behaviour is qualitatively similar for larger $\varepsilon$, with breakdown appearing for smaller $S_{c}$, e.g. $S_{c}=(0.65,0.58,0.50)$ for $\varepsilon=(0.15,0.25,0.50)$.

This collaborative research was supported by the Spanish Ministry of Science and Technology (MCyT) under Project\# DPI2002-04550-C07. A. R. also acknowledges the financial support of the MCyT through the Ramón y Cajal Program.

\section{REFERENCES}

Bell, J. B., Colella, P. \& Glaz, H. M. 1989 A second order projection method for the incompressible Navier-Stokes equations. J. Comput. Phys. 85, 257-283.

Beran, P. S. \& Culick, F. E. C. 1992 The role of non-uniqueness in the development of vortex breakdown in tubes. J. Fluid. Mech. 242, 491-527.

Buntine, J. D. \& Saffman, P. G. 1995 Inviscid swirling flows and vortex breakdown. Proc. R. Soc. Lond. A 449, 139-153.

Escudier, M. P. 1987 Confined vortices in flow machinery. Annu. Rev. Fluid Mech. 19, 27-52.

GöRTLER, H. 1954 Theoretical investigation of the laminar boundary layer. Problem II - decay of swirl in an axially symmetrical jet, far from the orifice. Report for United States Air Force, Office of Scientific Research, Contract No AF 61 (514)-627-C.

Guo, B., Langrish, T. A. G. \& Fletcher, D. F. $2001 a$ Numerical simulation of unsteady turbulent flow in axisymmetric sudden expansions. J. Fluids Engng 123, 574-587.

Guo, B., Langrish, T. A. G. \& Fletcher, D. F. $2001 b$ Simulation of turbulent swirl flow in an axisymmetric sudden expansion. AIAA J. 39, 96-102.

Hall, M. G. 1972 Vortex breakdown. Annu. Rev. Fluid Mech. 4, 195-218.

Herrada, M. A., Pérez-Saborid, M. \& Barrero, A. 2003 Vortex breakdown in compressible flows in pipes. Phys. Fluids 15, 2208-2218.

KIM, J. \& Morn, P. 1985 Application of a fractional-step method to incompressible Navier-Stokes equations. J. Comput. Phys. 59, 308-323.

Leibovich, S. 1978 The structure of vortex breakdown. Annu. Rev. Fluid Mech. 10, 221-246.

LoITSIANSKII, L. G. 1953 Propagation of a whirling jet in an infinite space filled with the same fluid. Prikl. Mat. Mekh. 17, 3-16 (in Russian).

Nathan, G. J., Hill, S. J. \& Luxton, R. E. 1998 An axisymmetric fluidic nozzle to generate jet precession. J. Fluid. Mech. 370, 347-380.

Nathan, G. J., Turns, G. J. \& Bandaru, R. V. 1996 The influence of fuel jet precession on the global properties and emissions of unconfined turbulent flames. Combust. Sci. Tech. 112, 211-230.

Patankar, S. V. \& Spalding, D. B. 1972 A calculation procedure for heat, mass and moment transfer in three-dimensional parabolic flows. Intl J. Heat Mass Transfer 15, 1787-1806.

Poinsot, T. \& Veynante, D. 2001 Theoretical and Numerical Combustion, pp. 269-280. R. T. Edwards.

Revuelta, A., Sánchez, A. L. \& Liñ́n, A. 2002a Confined axisymmetric laminar jets with large expansion ratios. J. Fluid Mech. 456, 319-352 (referred to herein as RSL).

Revuelta, A., SÁnchez, A. L. \& Liñán, A. $2002 b$ The virtual origin as a first-order correction for the far-field description of laminar jets. Phys. Fluids 14, 1821-1824.

Revuelta, A., SÁnchez, A. L. \& Liñán, A. 2004 The quasi-cylindrical description of submerged laminar swirling jets. Phys. Fluids 16, 848-851.

Ruith, M. R., Chen, P., Meiburg, E. \& Maxworthy, T. 2003 Three-dimensional vortex breakdown in swirling jets and wakes: direct numerical simulation. J. Fluid Mech. 486, 331-378.

Schlichting, H. 1933 Laminare strahlausbreitung. Z. Angew. Math. Mech. 13, 260-263.

Shtern, V. \& Hussain, F. 1999 Collapse, symmetry breaking \& hysteresis in swirling flows. Annu. Rev. Fluid Mech. 31, 537-66. 